\title{
A Cadaveric Study of Thread Cubital Tunnel Release with Newly Developed Threads
}

\author{
Minsuk Kang, ${ }^{1, *}$ Yong Seok Nam, ${ }^{2,3, *}$ In Jong Kim, ${ }^{4}$ Hae-Yeon Park, Jung Ryul Ham, ${ }^{5}$ Jae Min Kim ${ }^{6}$ \\ Department of Rehabilitation Medicine,' Seoul St. Mary's Hospital, College of Medicine, The Catholic University of Korea, Seoul, Korea \\ Department of Anatomy, Institute for Applied Anatomy, College of Medicine, The Catholic University of Korea, Seoul, Korea \\ Department of Anatomy, ${ }^{3}$ College of Korean Medicine, Dongshin University, Naju, Korea \\ Department of Rehabilitation Medicine, ${ }^{4}$ Howareyou Rehabilitation Clinic, Seoul, Korea \\ Department of Research \& Development, ${ }^{5}$ Ultra V Co. Ltd., Seoul, Korea \\ Department of Rehabilitation Medicine, Incheon St. Mary's Hospital, College of Medicine, The Catholic University of Korea, Seoul, Korea
}

Objective : The percutaneous thread transection technique is a surgical dissecting method using a dissecting thread inserted through a needle under ultrasound guidance without skin incision. As the new dissecting threads were developed domestically, this cadaver study was conducted to compare the effectiveness and safety between the new threads (ultra V sswire and smartwire-01) and a pre-existing commercial dissecting thread (loop \& shear) by demonstrating a modified looped thread cubital tunnel release.

Methods : The percutaneous cubital tunnel release procedure was performed on 29 fresh cadaveric upper extremities. The preexisting commercial thread was used in 5 upper extremities. The two newly developed threads were used in 24 upper extremities. Two practitioners performed the procedures separately. After the modified looped thread cubital release, anatomical and histological analyses were performed by a blinded anatomist. The presence of the dissected cubital tunnel and damaged adjacent soft tissue was assessed.

Results : Out of the 29 cadaveric upper extremities, 27 specimens showed complete dissection of the Osborne ligament and the proximal fascia of the flexor carpi ulnaris muscle. One specimen was incompletely dissected in each of the ultra V sswire and smartwire-01 groups. There were no injuries of adjacent structures including the ulnar nerve, ulnar artery, medial antebrachial cutaneous nerve, or flexor tendon with either the commercial thread or the newly developed threads. The anatomical analysis revealed clear and sharp incisional margins of the cubital tunnel in the Smartwire-01 and loop \& shear groups. All three kinds of threads maintained proper linear elasticity for easy handling during the procedure. The smartwire-01 provided higher visibility in ultrasound than the other threads.

Conclusion : The newly developed threads were effective and safe for use in the thread cubital tunnel release procedure.

Key Words : Cubital tunnel syndrome · Cadaver · Ultrasonography · Anatomy and histology.

- Received : June 1, $2021 \cdot$ Revised : July 10, $2021 \cdot$ Accepted : August 4, 2021

- Address for reprints : Jae Min Kim

Department of Rehabilitation Medicine, Incheon St. Mary's Hospital, College of Medicine, The Catholic University of Korea, 56 Dongsu-ro, Bupyeong-gu, Incheon 21431, Korea Tel : +82-32-280-5981, Fax : +82-32-280-5040, E-mail : jaeminmd@gmail.com, ORCID : https://orcid.org/0000-0003-4217-3228

*These authors contributed equally to this work.

This is an Open Access article distributed under the terms of the Creative Commons Attribution Non-Commercial License (http://creativecommons.org/licenses/by-nc/4.0) which permits unrestricted non-commercial use, distribution, and reproduction in any medium, provided the original work is properly cited. 


\section{INTRODUCTION}

Ulnar nerve entrapment is the second most common compressive peripheral neuropathy in the upper extremity. ${ }^{7)}$ The compression of the ulnar nerve at the elbow is known as cubital tunnel syndrome. The cubital tunnel is formed with retrocondylar groove and the Osborne ligament, a ligament spanning from the medial edpicondyle to the olecrenon process that connects with two heads of the flexor carpi ulnaris muscle, the most common site of ulnar nerve compression ${ }^{1)}$. A patient diagnosed with cubital tunnel syndrome will have complaints of sensory changes in half of the 4th finger and 5th finger, the ulnar side of the palm, and the ulnar side of the hand dorsum. As the lesion progresses, hand weakness can occur, and further progression may result in atrophy of the intrinsic muscles of the hand $d^{1)}$. Surgical treatment is required when initial conservative management fails to prevent progression over several months ${ }^{1)}$. Surgical treatment includes simple decompression of the nerve alone, decompression with ulnar nerve anterior transposition (subcutaneous, intramuscular, or submuscular), or medial epicondylectomy $y^{2,10)}$.

Using high-resolution ultrasound, the percutaneous dissecting thread technique was newly developed. The percutaneous thread transection technique is a surgical dissecting method whereby a dissecting thread is inserted through a needle under ultrasonography guidance without skin incision. Guo and colleagues conducted studies related to percutaneous dissecting thread techniques in several nerve compression syndromes including median, ulnar, and peroneal entrapment neuropathies ${ }^{4-6,8)}$.

The previous study of percutaneous cubital tunnel release was conducted by Guo et al. ${ }^{6}$ ) using a pre-existing commercial thread (loop \& shear; $0.23 \mathrm{~mm}$ in diameter; Ridge \& Crest Company, Monterey Park, CA, USA). However, the procedure was performed using a 2-stage dissection below and above the elbow. In contrast, the present study conducted the percutaneous cubital tunnel release procedure with a newly suggested 1-stage dissecting technique using newly developed threads. Two new domestic dissecting threads (ultra V sswire; Ultra V Co. Ltd., Seoul, Korea and smartwire-01; Smart Wire Co. Ltd., Ilsan, Korea) were developed for the percutaneous dissecting thread technique.

This cadaveric study was conducted to confirm the effectiveness and safety of the newly developed threads, to compare against the existing commercial thread, and to evaluate the modified technique of looped thread cubital tunnel release.

\section{MATERIALS AND METHODS}

As a result of contacting Catholic Medical Center (CMC) Office of Human Research Protection, we confirmed that this study is subject to exclusion of CMC Institutional Review Board as a cadaveric study that does not include personal identification information in accordance with human research determination of CMC Human Research Protection Program.

Fifteen (five males and 10 females) fresh cadavers with a mean age of $82.5 \pm 9.1$ years were used for this study. The percutaneous cubital tunnel release procedure was performed on 29 fresh cadaveric upper extremities. The cadaveric specimens were obtained through the Institute for Applied Anatomy. All cadaveric extremities were free from any evidence of trauma or surgery.

The newly developed threads are the ultra V sswire $(0.36$ $\mathrm{mm}$ in diameter; Ultra V Co. Ltd.) and the smartwire-01 (0.27 $\mathrm{mm}$ in diameter; Smart Wire Co. Ltd.). The smartwire-01 is an improved product of the ultra $\mathrm{V}$ sswire, thin and coated with titanium nitride. The commercial thread and the two newly developed threads consist of a similar chemical compo-

Table 1. Characteristics of the dissecting threads

\begin{tabular}{lccc}
\hline Thread & Loop \& shear & Ultra-V sswire & Smartwire-01 \\
\hline Chemical composition & $\mathrm{Fe}, \mathrm{Ni}, \mathrm{Cr}, \mathrm{Mo}$, Si, etc. & Fe, Ni, Cr, Mo, Si, etc. & Fe, Ni, Cr, Mo, Si, etc. \\
Material standard & STS316L & STS316L & STS316L \\
Wire diameter $(\mathrm{mm})$ & 0.23 & 0.36 & 0.27 \\
Maximum load $(\mathrm{N})$ & 31.5 & 74 & 42.4 \\
\hline
\end{tabular}

Fe : iron, Ni : nickel, Cr : chromium, Mo : molybdenum, Si : silicon, STS316L : stainless steel 316 and L denotes the low content of carbon 
sition and wire structure. However, the wire diameter and maximal load of each thread are different (Table 1). The maximal loadings of the newly developed threads are greater than that of the commercial thread, which means that the ultra $\mathrm{V}$ sswire and smartwire-01 are able to provide higher cutting force during the procedure than the commercial thread.

The pre-existing commercial thread (loop \& shear; 0.23 $\mathrm{mm}$ in diameter; Ridge \& Crest Company) was used in 5 upper extremities. Each of the newly developed threads (ultra $\mathrm{V}$ sswire and smartwire-01) was used in 12 upper extremities.

All procedures were performed using an RS85 ultrasound machine (Samsung Medison, Seoul, Korea) fitted with a L312A (3-12 MHz) linear array transducer, a compression setting based on the "musculoskeletal-elbow" factory preset. A highfrequency transducer was used to provide visualization of the cubital tunnel and the thread loop. The real-time ultrasound system was used to check the thread loop during the thread release procedure. Before performing the thread cubital tunnel release, ultrasound assessment of the cubital tunnel and ulnar nerve was completed. Two experienced practitioners performed the percutaneous cubital tunnel release procedures.

A schematic drawing illustrating the thread cubital tunnel release procedure is shown in Fig. 1. The cadaveric upper extremities were placed with pronation, which means olecranon faced upward, and the ultrasound was used in the procedure. Five $\mathrm{mL}$ of saline was used for hydrodissection of the ulnar nerve at the cubital tunnel. An 18-gauge Tuohy epidural needle connected with a $5 \mathrm{~mL}$ water-filled syringe was inserted at 4-8 $\mathrm{cm}$ proximal to the medial epicondyle where the ulnar nerve passes. The needle passed under the cubital tunnel with

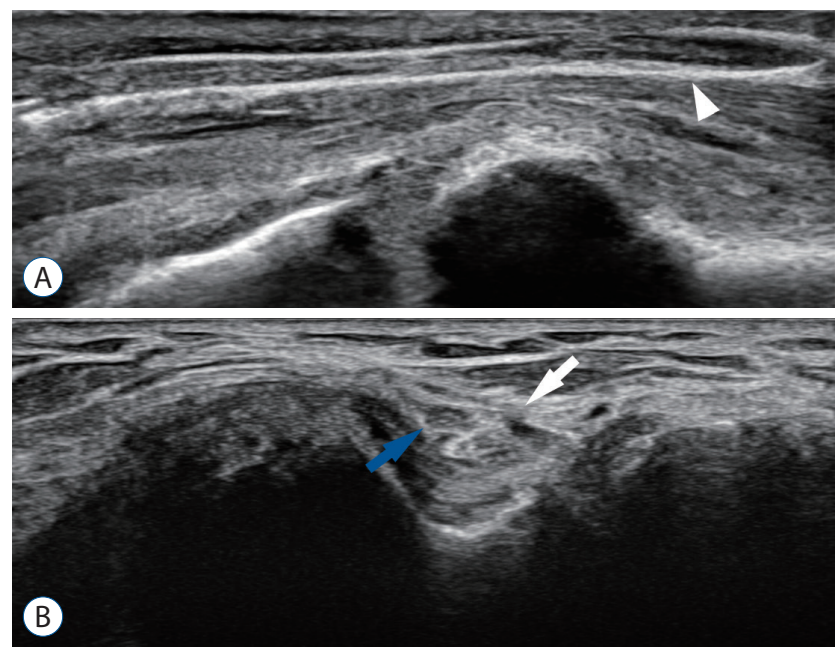

Fig. 2. Ultrasound pictures of the cubital tunnel. A : Long-axis view of the cubital tunnel. Arrowhead indicates the looped thread surrounding the Osborne ligament. B : Short-axis view of the cubital tunnel. Blue arrow indicates the ulnar nerve. White arrow indicates discontinuity of the cubital tunnel, dissected by the thread.
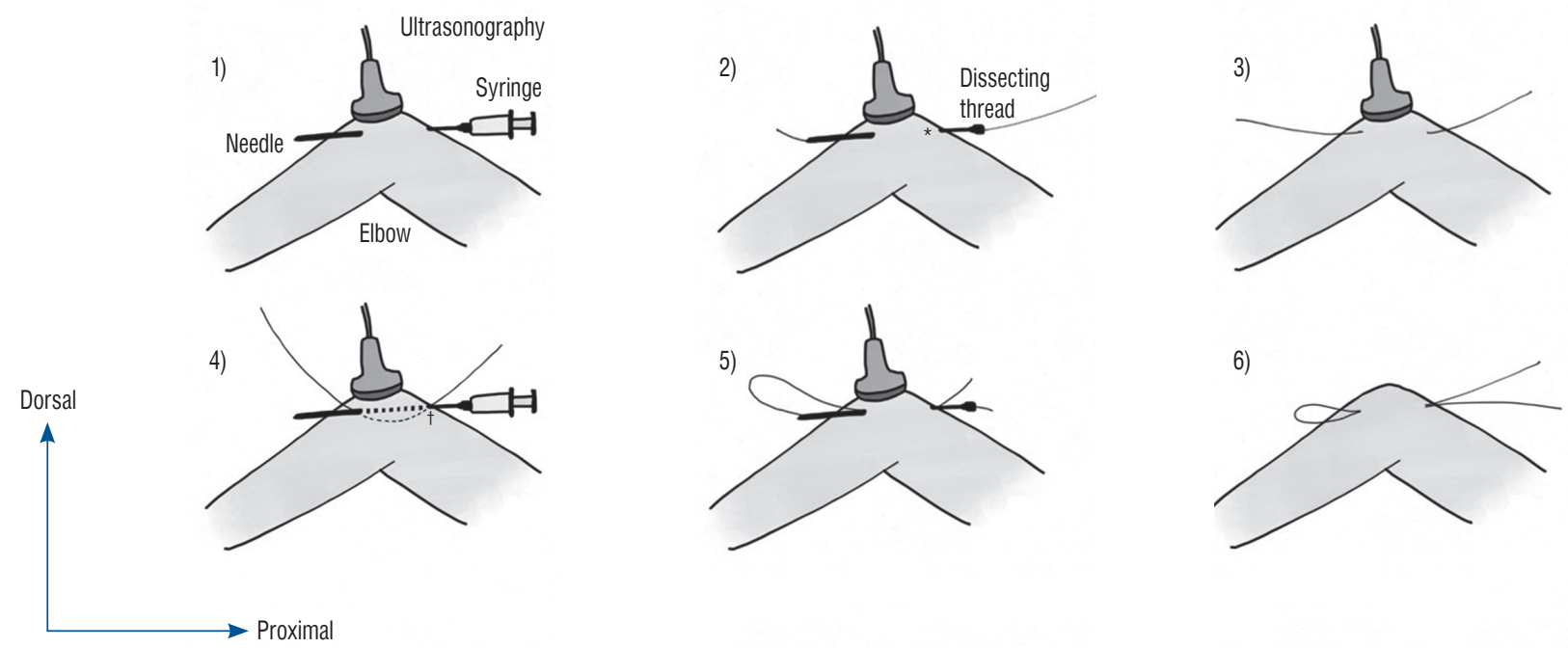

Fig. 1. Schematic drawing illustrating the percutaneous dissecting thread technique in the cubital tunnel release procedure. 1) The needle was inserted at the proximal to the medial epicondyle, passed under the cubital tunnel with hydrodissection around the nerve, and exited. 2) The dissecting thread passed through the needle. 3) The needle was removed, leaving the thread in the cubital tunnel. 4) The needle was reinserted at the same proximal entry point, passed above the cubital tunnel, and exited at the same distal point. 5) The distal dissecting thread, which remained in the cubital tunnel, was inserted into the distal needle tip. 6) After withdrawing the needle, the looped thread remained at the cubital tunnel. *Under cubital tunnel. ${ }^{\dagger}$ Above cubital tunnel. 
hydrodissection to create safe space around the nerve and exited at $4-8 \mathrm{~cm}$ distal to the medial epicondyle, passing through the Osborne ligament and the proximal fascia of the flexor carpi ulnaris muscle. The dissecting thread then passed through the needle and the needle was removed, leaving the thread in the cubital tunnel. With ultrasound guidance, the needle was reinserted at the same proximal entry point where the thread was inserted. The needle passed above the cubital tunnel and exited at the same distal point where the thread came out. The distal dissecting thread, which remained in the cubital tunnel, was inserted into the distal needle tip. After withdrawing the needle, the looped thread remained at the

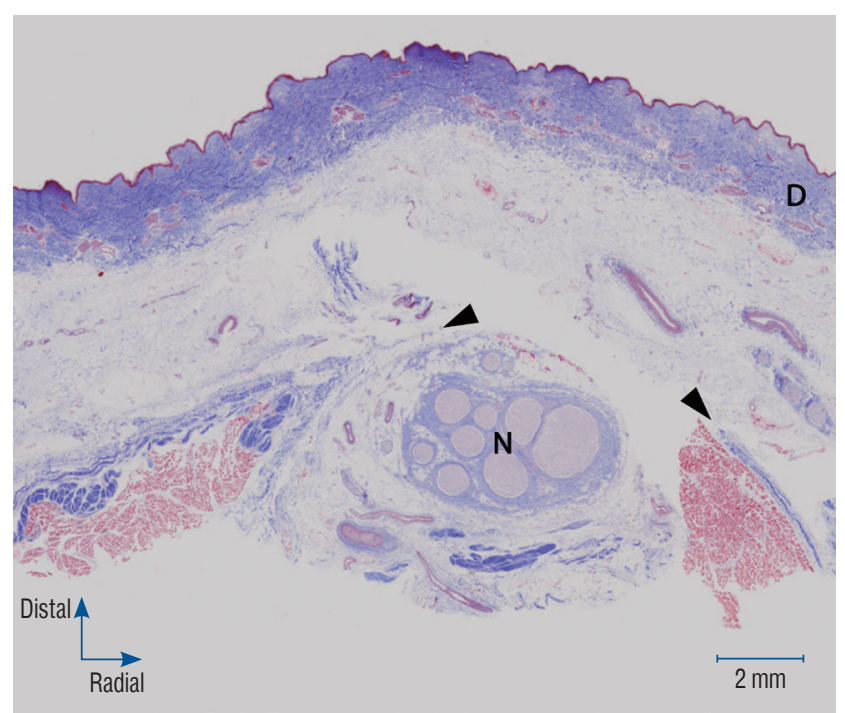

Fig. 3. Histologic photograph of the cross section of the dissected tissue with Masson's trichrome staining. Arrowheads indicate transected Osborne ligament. $\mathrm{N}$ indicates the ulnar nerve. $\mathrm{D}$ indicates dermis. cubital tunnel (Fig. 2A). The distal portion of the aponeurosis was transected by smooth traction with a reciprocating motion of the looped thread until the tread was pulled out.

The inlet of the cubital tunnel was defined as the triangular space bordered by the medial epicondyle, the olecranon, and the hyperechoic Osborne's band. The outlet of the tunnel was defined as the space bordered by the flexor carpi ulnaris heads, the ulna and the level where the two heads of the flexor carpi ulnaris joined. The thread loop was positioned include both the inlet and outlet of the cubital tunnel and gently transected the cubital tunnel.

After the thread cubital tunnel release, the analysis was performed by an anatomist with randomization. The cut parts were visually assessed by anatomical dissection to check for complete dissection of the cubital tunnel including the Osborne ligament and the proximal fascia of the flexor carpi ulnaris muscle. Additionally, a histological assessment was performed to determine the extent of damage, if any, to the ulnar nerve or other nearby structures by observing the cross sections of the dissected tissue with Masson's trichrome staining (Fig. 3).

To minimize injury to the Osborne ligament which covered the cubital tunnel, the antebrachial and brachial fascia, not the tendon of the flexor carpi ulnaris, were first identified with a magnifying glass, and investigation was performed with caudo-cranial direction following the fascia.

\section{RESULTS}

Five cadaveric upper extremities using the loop \& shear, 12

Table 2. The result of thread cubital tunnel release with loop \& shear, ultra V sswire, smartwire-01

\begin{tabular}{|c|c|c|c|}
\hline Cubital tunnel release & Loop \& shear $(n=5)$ & Ultra V sswire $(n=12)$ & Smartwire-01 $(n=12)$ \\
\hline \multicolumn{4}{|c|}{ Osborne's ligament \& proximal fascia of FCU } \\
\hline Unable to cut & $0(0.0)$ & $0(0.0)$ & $0(0.0)$ \\
\hline Partially cut & $0(0.0)$ & $1(8.3)$ & $1(8.3)$ \\
\hline Complete cut & $5(100.0)$ & $11(91.7)$ & $11(91.7)$ \\
\hline \multicolumn{4}{|l|}{ Damaged structures } \\
\hline Ulnar nerve & $0(0.0)$ & $0(0.0)$ & $0(0.0)$ \\
\hline MABC & $0(0.0)$ & $0(0.0)$ & $0(0.0)$ \\
\hline Ulnar artery & $0(0.0)$ & $0(0.0)$ & $0(0.0)$ \\
\hline Flexor tendon & $0(0.0)$ & $0(0.0)$ & $0(0.0)$ \\
\hline
\end{tabular}

Values are presented as number (\%). FCU : flexor carpi ulnaris muscle, MABC : medial antebrachial cutaneous nerve 
cadaveric upper extremities using the ultra V sswire, and 12 cadaveric upper extremities using the smartwire-01 were included in the data analysis.

Dissected Osborne ligaments with a small gap were observed in short-axis ultrasound view at the elbow (Fig. 2B). In all 29 cadaveric upper extremities including the loop \& shear, ultra V sswire, and smartwire-01 groups, complete dissections of the Osborne ligament and the proximal fascia of the flexor carpi ulnaris muscle were observed on visual assessment, with the exception of two specimens. One specimen with a partially dissected cubital tunnel was observed in each of the ultra $\mathrm{V}$ sswire and smartwire-01 groups (Table 2). No cross section or anatomical difference could be found between the two groups (Fig. 4). Incisional margins on the Osborne ligament were identified more sharply in the smartwire-01 (Fig. 4C) and loop \& shear groups (Fig. 4A) than in the ultra V sswire group (Fig. 4B). As confirmed by histologic evaluations, there were no injuries to the ulnar nerve, ulnar artery, medial antebrachial cutaneous nerve, or flexor tendon in any of the groups (Table 2). Furthermore, there were no observable skin injuries around needle penetrated sites, nor was there any difference between the two practitioners.

The newly developed domestic threads, ultra V sswire and smartwire-01, provided sufficient linear elasticity for handling and cutting force during the surgical procedure, comparable to those of the existing commercial thread, loop \& shear.
There were no significant differences in handling and cutting force during the procedure among the three groups, as the practitioners felt subjectively. However, the visibility of the Smartwire-01 in the ultrasound view was superior to that of
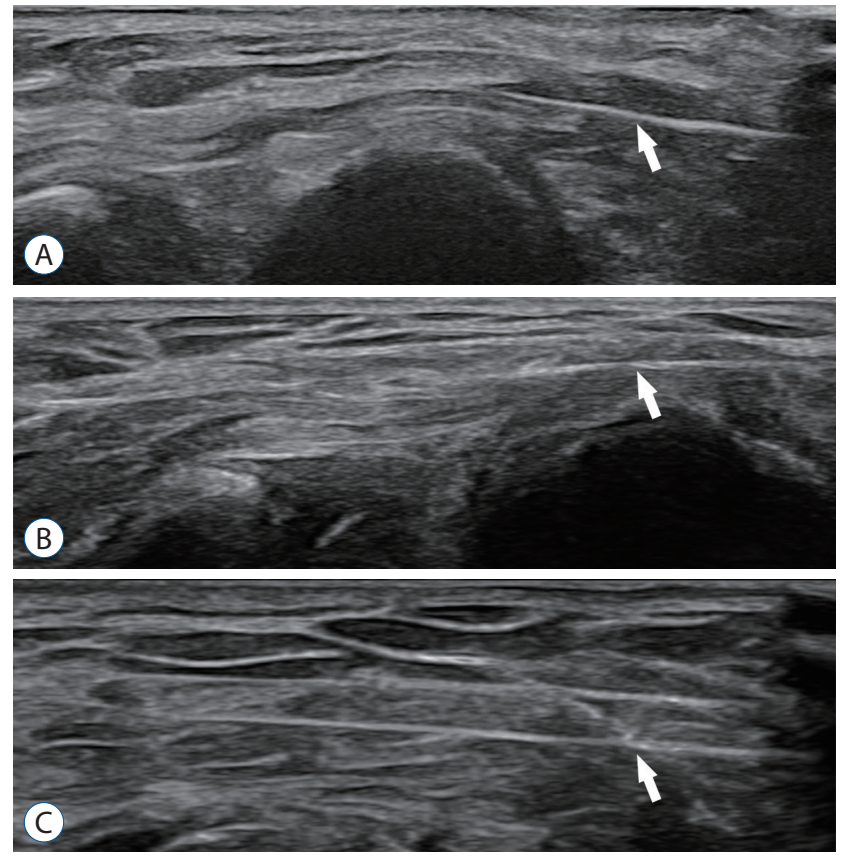

Fig. 5. Ultrasound findings of the long-axis view of the cubital tunnel. Arrow indicates the looped thread surrounding the Osborne ligament. Smartwire-01 was more visible in ultrasound view than other threads. A : Loop \& shear. B : Ultra V sswire. C : Smartwire-01.
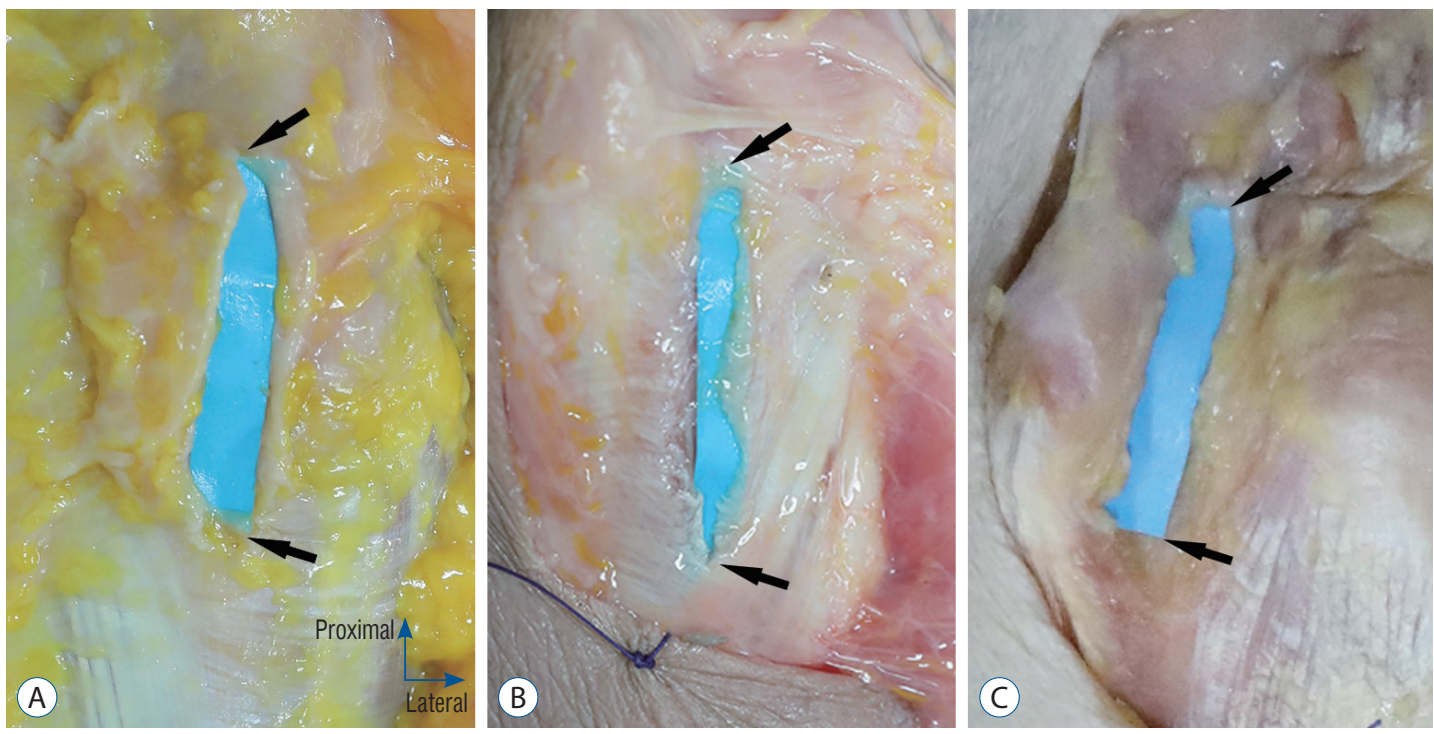

Fig. 4. Gross pictures of dissected elbows after performing the percutaneous dissecting thread technique in cubital tunnel release. Arrows indicated the proximal and distal end of the cubital tunnel. A : Dissected with loop \& shear. B : Dissected with ultra V sswire. C: Dissected with smartwire-01. 
the other two threads (Fig. 5).

\section{DISCUSSION}

A complete cubital tunnel release using dissecting thread was observed in all loop \& shear specimens and in 11 of the 12 specimens in each of the ultra $\mathrm{V}$ sswire and smartwire-01 groups. There was no nerve, vascular, or tendon damage observed in any of the 29 upper extremities. There were no significant differences in the dissection of the cubital tunnel or in damage to adjacent structures among the loop \& shear, ultra $\mathrm{V}$ sswire, and smartwire-01 threads. However, upon visual assessment, the incisional margins of the cubital tunnel after anatomical dissection were sharper in the loop \& shear and smartwire-01 groups than in the ultra $\mathrm{V}$ sswire group.

The newly developed domestic threads, ultra V sswire and smartwire-01, provided a cutting force during the procedure that was comparable to the existing commercial thread, loop \& shear. All three kinds of threads maintained proper linear elasticity for easy handling during the procedure. The Osborne ligament and the proximal fascia of flexor carpi ulnaris muscle dissected completely after 3-4 iterations of smooth reciprocating traction of the looped thread with all three kinds of threads. Although none of the threads in the three groups broke during the procedure, it can be expected that a newly developed thread with a higher maximum load value would be more stable for the procedure.

The visibility of the thread in ultrasound view was superior with the smartwire-01 thread compared to the other threads. Before the resection of the ligament, it is very important to identify whether or not any unwanted anatomical structures are included in the loop. Therefore, clear visibility of the thread in ultrasound view is a crucial aspect to take into consideration when selecting a dissecting thread. Unlike the existing commercial thread and the ultra V sswire, the smartwire-01 has a thin titanium nitride coating layer intended to provide additional cutting force and high visibility in ultrasound view. In this study, we could confirm the additional visibility of the smartwire- 01 in ultrasound view, provided by the thin titanium nitride coating layer (Fig. 5).

The smartwire-01 has several advantages in the percutaneous cubital tunnel release procedure. First, the smartwire- 01 is effective in dissecting the cubital tunnel without causing damage to the adjacent structures. Secondly, it provides as clear an incisional margin as the loop \& shear in the dissected cubital tunnel. Third, it has high visibility in ultrasound view and similar handling and cutting force during the procedure compared to the other threads, which contributes to the convenience and safety needed for the procedure. However, there were one partial cut species was observed in each ultra V sswire and smartwire-01 groups although new developed threads have high visibility in ultrasound image. It is considered that the partial cut was occurred due to technical error caused by status of cadaver limiting ultrasound images.

Previously mentioned operational techniques are effective in treating cubital tunnel syndrome; though, there are several complications. In open surgical procedure, disturbances to wound healing can occur with or without infection. Damage to the medial antebrachial cutaneous nerve and formation of perineural scars can occur. In addition, instability of the elbow joint after improper epicondylectomy or elbow joint contraction after long periods of immobilization is observed occasionally ${ }^{1,2,10}$. However, in percutaneous dissecting thread technique, by performing minimal incision, those are expected that decreased the risk of the damage to surrounding structures such as medial antebrachial cutaneous nerve, cosmetically better appearance and decreased scar discomfort due to reduced scar formation, and early return to work period with rapid recovery. Endoscopic cubital tunnel release is also effective treatement in cubital tunnel syndrome, which showed also minimal invasive technique ${ }^{3,9}$. Compared to the endoscopic cubital tunnel release, percutaneous dissecting thread technique is expected less scar formation and faster recovery time due to smaller incision. Moreover, vascular complications may be further reduced in percutaneous dissecting thread techniques because dopper image could visualize the blood flow around the cubital tunnel.

This new technique also has several limitations. The percutaneous dissecting thread technique is conducted under ultrasound guidance; therefore, the quality of the procedure depends on the ability of the operator. Direct nerve injury can also occur during the intervention. The structures related with cubital tunnel including ulnar nerve are not visually confirmed unlike open or endoscopic surgical prodecures. Additionally, there is no correction for nerve subluxation caused by this procedure. Further study including clincal trial is required to confirm the advantages and complications of 
the percutaneous dissecting thread technique.

The present cadaveric study has several strengths. The previous cadaveric study of percutaneous cubital tunnel release with a dissecting thread conducted by Guo and colleagues ${ }^{6}$ was performed using a 2-stage dissection, dissecting below and above the elbow. However, only Osborne ligament and deep fascia were evaluated in the study, not the release of arcade of Struthers, medial intermuscular septum or unintentional damaged structure around the proximal area. The present case conducted the procedure using a 1-stage dissection with successful transections of the Osborne ligament and the proximal fascia of flexor carpi ulnaris muscles, which is more effective compared to a 2-stage dissection. This procedure was possible because the new thread was clearly visible on the ultrasound image and easily distinguished from the surrounding structures, and it was possible to observe the procedure in real time. Two experienced experts performed the procedure separately, and one anatomist randomly analyzed the results, increasing the reliability of the results. Moreover, histologic evaluation as well as visual assessment was performed, increasing the accuracy of the results.

There are some limitations to this study. First, other than the Osborne ligament and the proximal fascia of flexor carpi ulnaris muscle, there has been no analysis of the procedure for common compression sites around the elbow, such as the arcade of Struthers or deep-flexor pronator aponeurosis. Second, the practitioners could not perform the procedure in a randomized fashion because due to the different shapes of the threads, they could easily notice which thread was being used.

\section{CONCLUSION}

Newly developed dissecting threads, ultra V sswire and smartwire-01, are as effective and safe as the pre-existing commercial thread, loop \& shear, in modified percutaneous cubital tunnel release.

\section{CONFLICTS OF INTEREST}

MK, YSN, IJK, and HYP declare no conflict of interests. JRH is an employee of Ultra V Co., Ltd. However, JRH reports non-financial support from Ultra $\mathrm{V}$ company, during the con- duct of the study. JMK reports grants from Catholic Medical Center, grants from National Research Foundation of Korea (NRF) grant funded by the Korean government (MSIT), during the conduct of the study. The new dissecting threads were made by Ultra V Co., Ltd. (\#804, ShinHan IT Tower, 19 Sangwon-gil, Seongdong-gu, Seoul, Korea) and Smart Wire Co., Ltd. (\#703, Moogoongwha-ro, Ilsan, Kyongki-do, Korea).

\section{INFORMED CONSENT}

This type of study does not require informed consent.

\section{AUTHOR CONTRIBUTIONS}

\author{
Conceptualization: JMK, YSN, IJK \\ Data curation : YSN, HYP, JMK \\ Formal analysis: YSN, HYP, MK \\ Funding acquisition : JMK \\ Methodology : JMK, YSN, IJK, JRH \\ Project administration : IJK, JRH, JMK \\ Visualization : MK, YSN \\ Writing - original draft : MK, YSN, IJK \\ Writing - review \& editing : MK, HYP, JMK
}

\section{ORCID}

$\begin{array}{ll}\text { Minsuk Kang } & \text { https://orcid.org/0000-0002-0468-1021 } \\ \text { Yong Seok Nam } & \text { https://orcid.org/0000-0001-8698-639X } \\ \text { In Jong Kim } & \text { https://orcid.org/0000-0002-7143-6114 } \\ \text { Hae-Yeon Park } & \text { https://orcid.org/0000-0002-7773-6329 } \\ \text { Jung Ryul Ham } & \text { https://orcid.org/0000-0003-2724-020X } \\ \text { Jae Min Kim } & \text { https://orcid.org/0000-0003-4217-3228 }\end{array}$

\section{- Acknowledgements}

This work was supported by the National Research Foundation of Korea (NRF) grant funded by the Korean government (Ministry of Science and Information \& Communications Technology) (No. NRF-2020R1F1A1055076). URLs: https:// www.nrf.re.kr/eng/index. The authors wish to acknowledge the financial support of the Catholic Medical Center Research 
Foundation made in the program year of 2020.

\section{References}

1. Andrews K, Rowland A, Pranjal A, Ebraheim N : Cubital tunnel syndrome: anatomy, clinical presentation, and management. J Orthop 15 : 832-836, 2018

2. Boone $S$, Gelberman RH, Calfee RP : The management of cubital tunnel syndrome. J Hand Surg Am 40 : 1897-1904; quiz 1904, 2015

3. Buchanan PJ, Chieng LO, Hubbard ZS, Law TY, Chim H : Endoscopic versus open in situ cubital tunnel release: a systematic review of the literature and meta-analysis of 655 patients. Plast Reconstr Surg 141 : 679-684, 2018

4. Guo D, Guo D, Guo J, Schmidt SC, Lytie RM : A clinical study of the modified thread carpal tunnel release. Hand (N Y) 12 : 453-460, 2017
5. Guo D, Guo D, Harrison R, McCool L, Wang H, Tonkin B, et al. : A cadaveric study using the ultra-minimally invasive thread transection technique to decompress the superficial peroneal nerve in the lower leg. Acta Neurochir (Wien) 161 : 2133-2139, 2019

6. Guo D, Kliot M, McCool L, Senk A, Tonkin B, Guo D : Percutaneous Cubital tunnel release with a dissection thread: a cadaveric study. J Hand Surg Eur Vol 44 : 920-924, 2019

7. Kim JH, Won SJ, Rhee WI, Park HJ, Hong HM : Diagnostic cutoff value for ultrasonography in the ulnar neuropathy at the elbow. Ann Rehabil Med 39 : 170-175, 2015

8. McCool L, Guo D, Guo D, Harrison R, Tonkin B, Senk A, et al. : Thread common peroneal nerve release-a cadaveric validation study. Acta Neurochir (Wien) 161 : 1931-1936, 2019

9. Smeraglia F, Del Buono A, Maffulli N : Endoscopic cubital tunnel release: a systematic review. Br Med Bull 116 : 155-163, 2015

10. Staples JR, Calfee R : Cubital tunnel syndrome: current concepts. J Am Acad Orthop Surg 25 : e215-e224, 2017 